\title{
Geometrical Feature Extraction Using 2D Range Scanner
}

\author{
Sen Zhang Lihua Xie Martin Adams Fan Tang \\ BLK S2, School of Electrical and Electronic Engineering \\ Nanyang Technological University, Singapore 639798 \\ Email: elhxie@ntu.edu.sg
}

\begin{abstract}
In this paper, two new algorithms for feature detection are presented. The first one can detect edge and circle features accurately using the Gaussian-Newton optimization method to fit the circle parameters. It consists of two parts: the first is the segmentation of data of each scan which is followed by parameter acquisition. The algorithm is off-line in nature as the segmentation and parameter acquisition are carried out after each scan data is collected. We also present another algorithm which is on-line and applies a multiple models filtering approach to handle features of different geometries such as lines and circles. It detects the circle features using the unscented Kalman filter. Experimental results show that the proposed two approaches are efficient in detecting features.
\end{abstract}

\section{Introduction}

One of the key problems of navigation is the reliable acquisition of information from the sensor. To address this issue, an appropriate representation of mobile robot's working environment should be acquired. A very popular approach is based on features [4]. Lots of navigation systems use artificial beacons to realize their navigation task, but the approach may not be realistic in large scale environment. In this situation, one needs to utilize the naturally occurring structure of typical environments to achieve a similar performance. Hence, reliable algorithms capable of extracting features from a large set of noisy data are important.

Some of the early efforts in this direction have focused on extracting line features in an indoor environment. In [1], a recursive line fitting system is used to extract line segments under polar coordinates and an ellipse fitting method is also implemented for data from a laser sensor. In [2], a regression least-squares parameter estimation was applied to detect line segments and the center and radius of a circle feature only using the average value of the measurements of the circle from a $2 \mathrm{D}$ range scanner. Instead of fitting straight line segments after a full scan has been recorded, Adams presented an on-line edge extraction approach employing a Kalman filter in [3]. Later, a two-layer Kalman filter is used to calculate the parameters of a line by an on-line means in [6]. All these methods are focused on indoor applications and line extraction. For an outdoor environment, the problem of feature selection and detection still remains. In our view, in most typical semi-structured outdoor environments, such as campus, parks, suburb roads, tree trunks and tree-like objects, such as pillars, are relatively stable, regular and naturally occurring features, and can provide useful information for future navigation. Recently, some research on the use of these kinds of geometrical features has been carried out in [8]. Also, [4] addressed the problem of extracting tree trunks from laser scan data.

In this paper, we propose two approaches to the circle feature and edge feature extraction. Currently, there are few papers focused on this problem. The existing methods for circle detection can only estimate the circle parameters roughly by a batch calculation, therefore the results are very sensitive to the measurements of large errors. The proposed two new methods are more robust and more accurate in detecting the features and acquiring the feature parameters. It should be noticed that these methods are also suitable for the cluster circle detection in indoor environment [2].

\section{Gauss-Newton Extraction Algorithm}

The essential components of this algorithm includes two parts: the first is the segmentation of the scan data and the second is the parameter acquisition. During the data segmentation process, only large discontinuities of the measurement data are identified so as to divide the scan data into several groups. For each group of data, we shall decide whether it is associated with a plane surface or a cylindrical object. For the latter we use the circle fitting algorithm to obtain parameters of the object.

\subsection{Data Segmentation}

Segmentation is a process of aiming to classify each scan data into several groups, each of which possibly 
associates with different structures of surrounding. The segmentation process is realized through EKF [3]. At each time instant the range estimate is compared to the range measurement based on their statistics in order to decide if an edge has been detected. When the difference between the measured range and the predicted range is beyond certain threshold, we consider that an edge has been detected. This can be achieved by using a validation gate during the prediction process with the EKF. This will be discussed in Section 2.1.3 in detail.

Consider a vertical plane shown in Fig.1. It is possible to show that the relationship between successive range readings, when the light beam is incident upon the surface, is given by [3] [9]

$$
d_{i+2}=\frac{d_{i} d_{i+1}}{2 d_{i} \cos \gamma-d_{i+1}}
$$

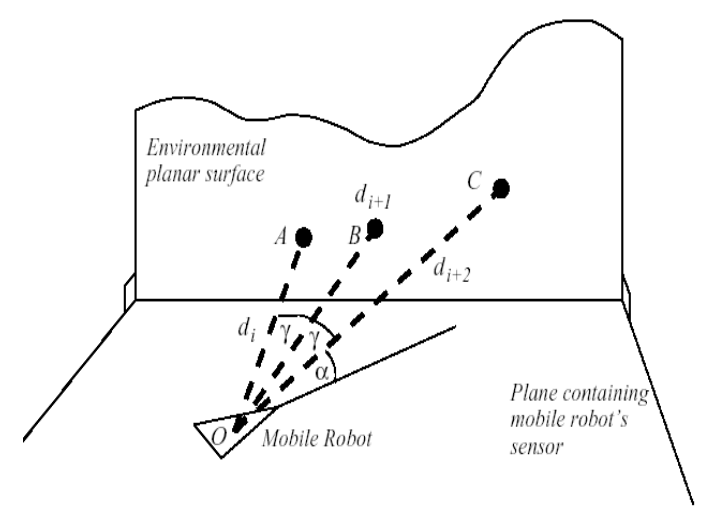

Figure 1: the relationship between successive range readings when scanning a planar surface

\subsubsection{System Model: Define}

$$
\begin{aligned}
& x_{1}(k+1)=d_{i+2} \\
& x_{2}(k+1)=x_{1}(k)=d_{i+1}
\end{aligned}
$$

where $x_{1}(k)$ and $x_{2}(k)$ are the state variables. Therefore equation (2.1) can be fully defined by the state space equations:

$$
x(k+1)=f(x(k))+v(k)
$$

where $x(k+1)=\left[\begin{array}{ll}x_{1}(k+1) & x_{2}(k+1)\end{array}\right]^{T}$, $f(x(k))=\left[\begin{array}{c}\frac{x_{1}(k) x_{2}(k)}{\left(2 x_{2}(k) \cos \gamma-x_{1}(k)\right)} \\ x_{1}(k)\end{array}\right]$ and $v$ is a white noise with a given small variance $Q(k)$ which implies that the surface is close to be perfect.

2.1.2 Observation Model: Since there is almost no angular uncertainty for the Sick sensor, we only consider the range measurement. The observation model is:

$$
z(k)=H x(k)+\omega(k)
$$

where $H=\left[\begin{array}{ll}1 & 0\end{array}\right], x(k)$ is the same as in (2.4) and $\omega(k)$ is a zero mean Gaussian noise with a known variance $\sigma_{r}^{2}$. By studying the statistics of the range measurement of the laser scanner, we found that the sensor noise follows approximately a Gaussian distribution with standard deviation ranging from 0.015 to 0.020 meter. We thus take the variance to be $\sigma_{r}^{2}=0.00040$.

The EKF is used to realize the prediction and validation process as in [3].

2.1.3 Validation Gate: In order to identify if a measurement is associated with a new edge, certain criterion needs to be established. Use the innovation $\nu(k+1)=z(k+1)-\hat{z}(k+1)$ and its variance $s(k+1)$ during the EKF process to define:

$$
d(k+1)=\nu^{T}(k+1) s^{-1}(k+1) \nu(k+1)
$$

Note that $d$ is a random variable following the $\chi^{2}$ distribution. A validation gate, $\delta$, is used to decide whether the measurement is a close enough match to the predicted data point to continue the filter update. If the measurement is such that $d(k+1)>\delta$, a discontinuity is found. From the $\chi^{2}$ distribution table, we know that if the observation is from the same planar surface, then $d(k+1)<9$ with a probability of 0.997 . If a small $\delta$ is selected, there will be more edges found. In our application, the validation gate can be set to be large to tolerate those measurements that don't match the prediction very well, thus only the large discontinuities can be detected. Here we set $\delta=9$.

After the data segmentation, we need to decide if each segment of data is associated with a line or an arc. For a line prediction, the average error between the observation and the prediction at each point should be very small. Note that the measurement error of the Sick sensor is a Gaussian noise with zero mean and variance of $\sigma_{r}^{2}$. Therefore, the average error $\bar{e}(n)$ of $n$ samples follows the Gaussian distribution of zero mean and variance of $\sigma_{r}^{2} / n$. Hence, $P\left\{|\bar{e}(n)| \leq 3 \sigma_{r} / \sqrt{n}\right\}=0.997$ For example, if $n=100$, then the probability $P\{|\bar{e}(n)| \leq 0.006\}=0.997$. since $\sigma_{r}=0.02$. By the study of samples, the threshold for the average error can be chosen to be $0.01 \mathrm{~m}$. If the average error is smaller than 0.01, we consider that this segment is associated with a line, otherwise, it is associated with an arc. For an arc, the Gaussian-Newton method [7] is applied to obtain its center and radius.

\subsection{Parameter Acquisition}

A circle can be defined by the equation

$$
\left(x-x_{0}\right)^{2}+\left(y-y_{0}\right)^{2}=r^{2}
$$

where $\left(x_{0}, y_{0}\right)$ and $r$ are the center and the radius of the circle, respectively. For a circle fitting problem, the data set $(x, y)$ is known and the circle parameters need 
to be estimated. Assume that we have obtained $M$ measurements $\left(x_{m}, y_{m}\right)$, Our objective is to find $p=$ $\left(x_{0}, y_{0}, r\right)$ that minimize

$$
E(p)=\sum_{m=1}^{M}\left[\left(x_{m}-x_{0}\right)^{2}+\left(y_{m}-y_{0}\right)^{2}-r^{2}\right]^{2}
$$

This is a typical nonlinear least-squares problem. We propose to use the modified Gauss-Newton method to solve the problem. In our case the Jacobian matrix for the modified Gaussian-Newton algorithm is

$$
A=\left[\begin{array}{ccc}
\frac{\partial f_{1}}{\partial x_{0}} & \frac{\partial f_{1}}{\partial y_{0}} & \frac{\partial f_{1}}{\partial r} \\
\frac{\partial f_{2}}{\partial x_{0}} & \frac{\partial f_{2}}{\partial y_{0}} & \frac{\partial f_{2}}{\partial r} \\
\vdots & \vdots & \vdots \\
\frac{\partial f_{M}}{\partial x_{0}} & \frac{\partial f_{M}}{\partial y_{0}} & \frac{\partial f_{M}}{\partial r}
\end{array}\right]
$$

Let $\bar{f}=\left(f_{1} f_{2} \ldots f_{M}\right)^{T}$ with

$f_{m}\left(x_{0}, y_{0}, r\right)=\left(x_{m}-x_{0}\right)^{2}+\left(y_{m}-y_{0}\right)^{2}-r^{2}$. At the $k$-th step, using the modified Gauss-Newton method to search the solution according to:

$$
\left(A_{k}^{T} A_{k}+\lambda_{k} I\right) \triangle p_{k}=-A_{k}^{T} \bar{f}_{k}
$$

where $\triangle p_{k}=p_{k+1}-p_{k}$ and $p_{k}$ is the estimate of $p=$ $\left[\begin{array}{lll}x_{0} & y_{0} & r\end{array}\right]^{T}$ at the $k$-th iteration.

We set the initial value $\lambda_{0}=0.01$ and carry out the following iterations for calculating an suboptimal $p$ :

Step 1: Calculate $\triangle p_{k}$ using equation (2.10);

Step 2: Calculate the sum error $E\left(p_{k}+\triangle p_{k}\right)$ by equation (2.8);

Step 3: Compare with the sum error of last step $E\left(p_{k}\right)$, if $E\left(p_{k}+\triangle p_{k}\right)>E\left(p_{k}\right)$, increase $\lambda_{k}$ by a factor of 10 , and go back to Step 1;

step 4: If $E\left(p_{k}+\triangle p_{k}\right)<E\left(p_{k}\right)$, decrease $\lambda_{k}$ by a factor of 10 , replace $p_{k}$ by $p_{k}+\triangle p_{k}$ and go back to Step 1 until the algorithm converges.

A starting guess for these parameters are required. The initial value of $\left(x_{0}, y_{0}, r\right)$ is got by using the first three points $\left(x_{i}, y_{i}\right) \quad i=1,2,3$. To demonstrate the applicability of the algorithm, we generate 10 noise data sets $\left(x_{i}, y_{i}\right) \quad i=1, \ldots 10$ from the circle $(x-5)^{2}+(y-10)^{2}=0.5^{2}$ to form an arc. Using the Gauss-Newton method to fit the arc. The results is: $x_{0}=4.9966, y_{0}=9.9933, r=0.4957$. The algorithm converges after 7 iterations.

\section{Multi-Model Filtering Extraction Approach}

Observe that the Gaussian-Newton method is an offline method. In this section, we shall consider an online multi-model (line model and circle model) filtering approach. Recall that the line model has been given in Section 2. In the following we shall establish a circle model and propose a tracking method for this model.

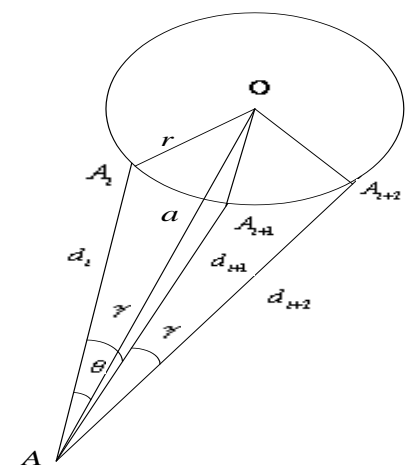

Figure 2: the relationship between successive range readings when scanning a cylindrical surface

\subsection{Circle Model and Its Tracking Algorithm}

Consider a circle arc that is recorded when the 2D laser scans a cylindrical object shown in Fig. 2. The laser beam originating from $A$ intersects the object at the consecutive points $A_{i}, A_{i+1}$, and $A_{i+2}$. The distances between $A$ and these points are $d_{i}, d_{i+1}$ and $d_{i+2}$, respectively. The circle has a center at point $o$ whose coordinate is $\left(x_{0}, y_{0}\right)$ in a local Cartesian coordinate frame (the origin is at point $A$ ) and the radius is $r$.

3.1.1 System Model: From Fig. 2, we can show that the relationship among range readings, when the light beam is incident upon the cylindrical surface, is given by

$$
\left\{\begin{array}{l}
r^{2}=d_{i+1}^{2}+a^{2}-2 d_{i+1} a \cos (\gamma-\theta) \\
r^{2}=d_{i+2}^{2}+a^{2}-2 d_{i+2} a \cos (2 \gamma-\theta) \\
a^{2}=x_{0}^{2}+y_{0}^{2}
\end{array}\right.
$$

From equation (3.1), we can obtain:

$d_{i+2}=\sqrt{x_{0}^{2}+y_{0}^{2}}(\xi-\eta)-\sqrt{\left(x_{0}^{2}+y_{0}^{2}\right)(\xi-\eta)^{2}-x_{0}^{2}-y_{0}^{2}+r^{2}}$

where $\xi=\frac{\cos \gamma\left(d_{i+1}^{2}+x_{0}^{2}+y_{0}^{2}-r^{2}\right)}{2 d_{i+1} \sqrt{x_{0}^{2}+y_{0}^{2}}}$;

$\eta=\frac{1}{2} \sin \gamma \sqrt{4-\frac{\left(d_{i+1}^{2}+x_{0}^{2}+y_{0}^{2}-r^{2}\right)^{2}}{d_{i+1}^{2}\left(x_{0}^{2}+y_{0}^{2}\right)}}$.

Let the state variables be: $x_{1}(k)=x_{0} ; \quad x_{2}(k)=$ $y_{0} ; \quad x_{3}(k)=r ; \quad x_{4}(k)=d_{i+1}$, then, we have:

$$
\left\{\begin{array}{l}
x_{1}(k+1)=x_{1}(k) \\
x_{2}(k+1)=x_{2}(k) \\
x_{3}(k+1)=x_{3}(k) \\
x_{4}(k+1)=d_{i+2}=g\left(x_{1}(k), x_{2}(k), x_{3}(k), x_{4}(k)\right)
\end{array}\right.
$$

The above can be put into the general form:

$$
x(k+1)=f(x(k))+v(k)
$$

where $x=\left[\begin{array}{llll}x_{1} & x_{2} & x_{3} & x_{4}\end{array}\right]^{T}$,

$$
f(x(k))=\left[\begin{array}{c}
x_{1}(k) \\
x_{2}(k) \\
x_{3}(k) \\
g\left(x_{1}(k), x_{2}(k), x_{3}(k), x_{4}(k)\right)
\end{array}\right] \text { and } v(k) \text { is }
$$


a noise vector introduced to reflect the imperfection of the cylindrical surface.

3.1.2 Observation Model: Our observation model is:

$$
z(k)=\left[\begin{array}{llll}
0 & 0 & 0 & 1
\end{array}\right] x(k)+\omega(k)
$$

where $x(k)$ is the same as that in equation $(3.4), \omega(k)$ is a zero mean noise with a known variance $\sigma_{r}^{2}$.

Note that the state equation (3.4) is highly nonlinear and complicated, which would lead to a complicated Jacobian matrix if an EKF is to be applied. Realizing this difficulty, we use the unscented Kalman filter(UKF) to perform the prediction and validation process as [5]. Here the equations of the UKF are omitted.

\subsection{Multiple Models Tracking}

In the above, we have presented a tracking method for objects of cylindrical shape together with the line detection method in Section 2.1. They form a multiple model feature extraction approach. It can be carried out as follows. Assuming that we start from a line model. The tracking method in Section 2.1 can be applied. The EKF can give a predicted distance for each newly arrived point and we can decide if the measurement is close enough to the predicted one by applying the validation gate as explained in section 2.1.3. When a discontinuity is found, the straight line segment being tracked has finished. Then filtering based on a new model (line or circle) is initialized. Note that the filter initialization uses the first three measurements after the discontinuity to calculate the center and the radius of a assumed circle. If the radius is large(greater than 0.8 meter), we consider that the next segment is also a line or cluster that is not the circle feature. So we start tracking based on a new line model until the next discontinuity. On the other hand, if the radius is smaller than a threshold(here using $0.8 \mathrm{~m}$ ), we think the next point group may represent a circle or a circle shape cluster and initialize the filtering based on a circle model that is introduced in the last section. The process is performed until the next discontinuity. The criterion for discontinuity in the second filter is the same as that in the first one(validation gate). It is noted that here a threshold 0.8 meter is used because usually the tree trunks's radius is smaller than 0.8 meter.

\section{Experimental Results}

The laser used in this experiment is Sick PLS with accuracy of $50 \mathrm{~mm}$. The field of view is 180 degrees and up to 50 meters of distance. To obtain a 360 degrees scene, we divide one whole scan in a particular position into 2 steps. First, the robot scans the scene in front of it, then turns around to sense the remaining 180 degrees scene. Thus we can acquire the 360 degrees sight for each location. The range samples are spaced every

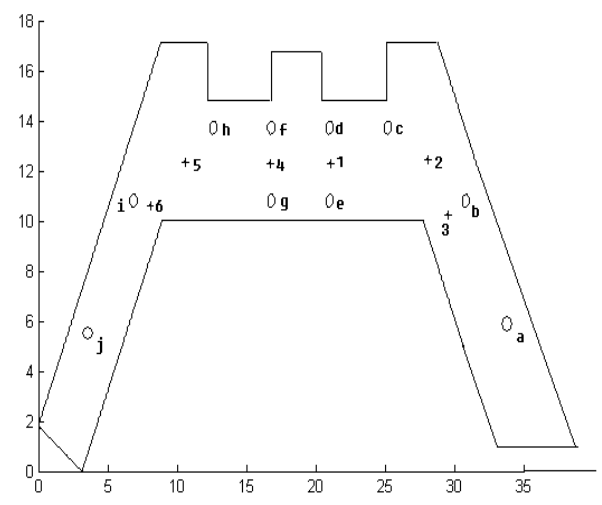

Figure 3: The platform to be explored by the robot.

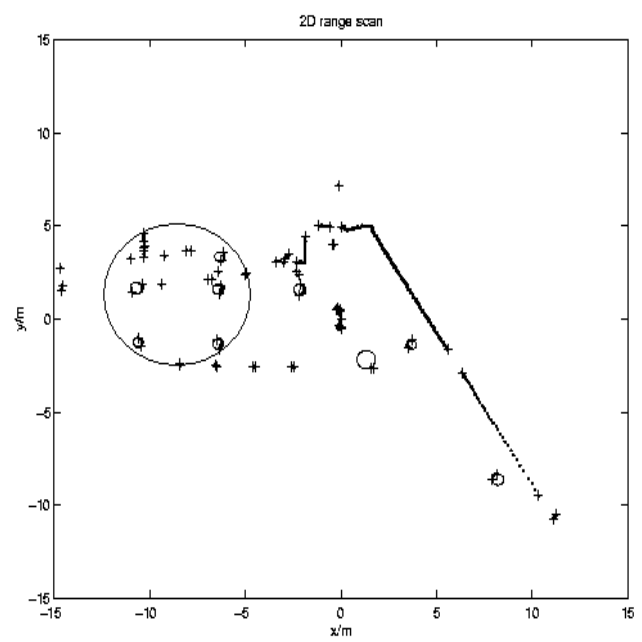

Figure 4: Circles and edges extracted from data at position 2 using the proposed Gaussian-Newton method

half a degree, all within the same plane. Experiment data is collected from a place outside a building where there are 10 pillars in Fig. 3 from $a$ to $j$, and the surroundings are building walls and low balusters. In this figure, the six cross points represent the different positions at which the robot scans the surroundings. We have done the feature detection for all the 6 laser scans. Since the scan data is similar, we only show the feature extraction results at position 2. The results are given in Fig. 4 to Fig. 7. Because the whole scan is about 50 meters at some points, the circle features cannot be seen clearly, we only show parts of the area which includes the pillars. Both the algorithms may result in additional circles found. This will affect navigation because in other scans these circles may not be detected. Further studies on this aspect are needed. 


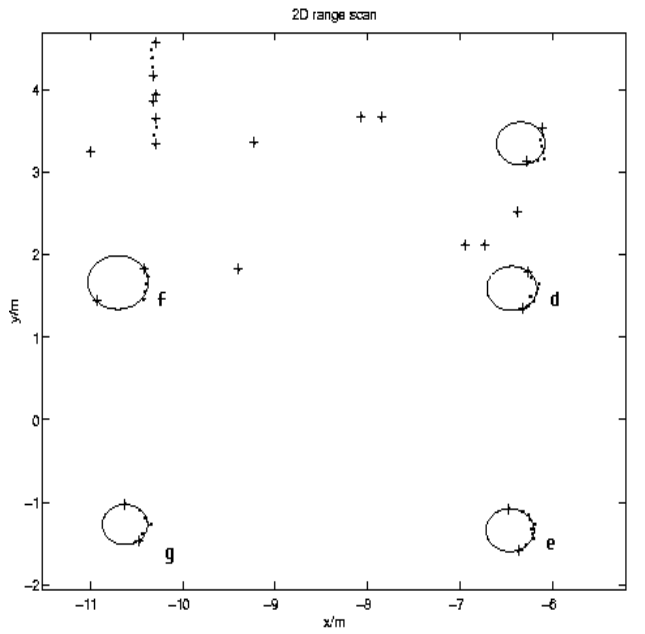

Figure 5: A zoomed view of the region inside the big circle in Fig. 6

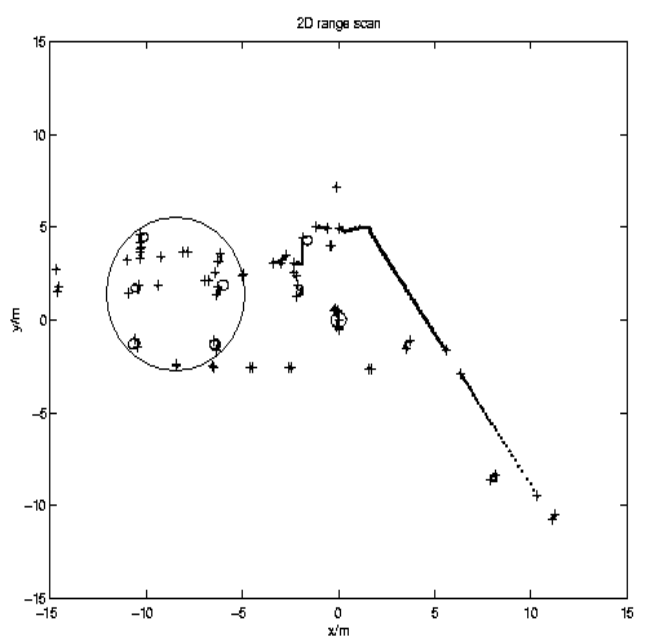

Figure 6: Circles and edges extracted from data at position 2 using the multiple models filtering method

\section{Conclusions}

In this paper two new algorithms for feature detection in outdoor environment have been presented. They can be used for the extraction of planar surfaces and tree trunks or tree-like objects in outdoor environment for mobile robots navigation. The off-line circle detection based on the Gaussian-Newton algorithm is very accurate in tracking circle and estimating feature parameters. The on-line multiple models filtering method is able to perform reasonably well even though it is not as accurate as the first algorithm. Experiment results show that both the methods can extract most of the features. They, however, also output features which do not physically exist. The problem of minimizing the number of physically non-existence features deserves further investigations.

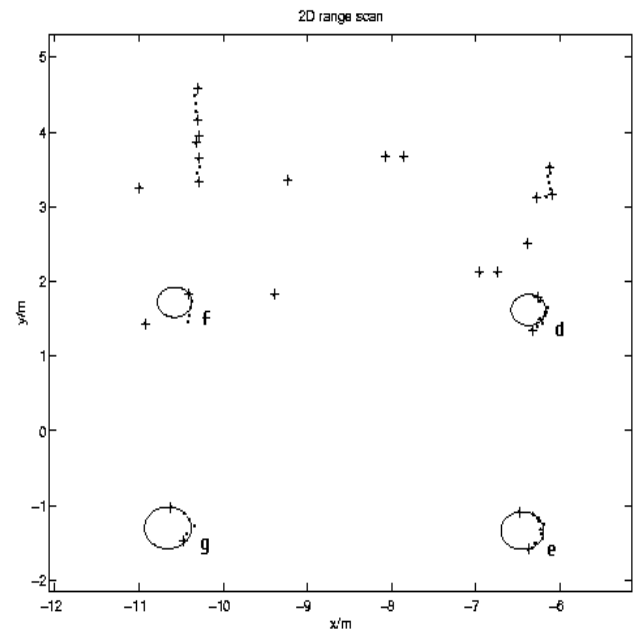

Figure 7: A zoomed view of the region inside the big circle in Fig. 8

\section{References}

[1] R. M. Taylor., P. J. Probert., "Range finding and feature extraction by segmentation of images for mobile robot navigation," In Proc. of the IEEE International Conference on Robots and automation, April, 1996.

[2] J. Vandorpe, H. V. Brussel, H. Xu, "Exact dynamic map building for a mobile robot using geometrical primitives produced by a 2D range finder," In Proc. of the IEEE International Conference on Robots and Automation, April, 1996.

[3] M. D. Adams., A. Kerstens., "Tracking natrurally occuring indoor features in $2 \mathrm{D}$ and $3 \mathrm{D}$ with Lidar Range/Amplitude data," International Journal of Robotics Research, 17(9), September 1998, pp907-923.

[4] T. Bailey., "Mobile robot localization and mapping in extensive outdoor enviroment" Ph.D Thesis, 2002 .

[5] S. Julier, J. Uhlmann, H. F. Durrant-Whyte, "A new method for the nonlinear transformation of means and covariances in filters and estimators," IEEE Transactions on Automatic Control, 2000.

[6] S. I. Roumeliotis, G. A. Bekey, "Segments: a Layered, dual Kalman filter algorithm for indoor feature extraction," Proceedings of the 2000 IEEE/RSJ International Conference on Intelligent Robots and Systems, pp454-461.

[7] J. Nocedal, S. J. Wright, "Numerical optimization," Springer Verlag, 1999

[8] J. Guivant, E. M. Nebot, S. Baiker, "Localization and map buiding using laser range sensors in outdoor applications," Journal of Robotics Systems, 17(10): pp565-583 2000.

[9] M. D. Adams., "Sensor modeling, design and data processing for autonomous navigation" World Sentific, 1999 